EESTI NSV TEADUSTE AKADEEMIA TOIMETISED. 25. KOIDE FUUSIKA * MATEMAATIKA. 1976, NR. 1

ИЗВЕСТИЯ АКАДЕМИИ НАУК ЭСТОНСКОИ ССР. ТОМ 25 ФИЗИКА * МАТЕМАТИКА. 1976, № 1

\title{
О ВЗАИМОСВЯЗИ АПОДИЗАЦИИ И РАЗРЕШАЮЩЕЙ СПОСОБНОСТИ
}

Ослабление побочных максимумов (аподизация) в диффракционной картине, полу ченной для точечного источника света с помощью оптического прибора, исследовалось многими авторами (см., напр., $\left[{ }^{1,2}\right]$ и др.). В меньшей степени изучалось аналитически сужение центрального максимума, т. е. повышение разрешающей способности в обычном смысле. Взаимосвязь между обеими этими характеристиками остается до сих пор почти неизученной (не считая попыток в $\left[{ }^{1-3}\right]$ ).

В данной работе мы применим для выяснения взаимосвязи между названными характеристиками свойства амплитудно-фазовых фильтров.

Известно $\left[{ }^{2}\right]$, что если круглое входное отверстие (с радиусом $a=1$ ) оптического прибора закрыто $n$-компонентным амплитудным фильтром, то распределение амплитуды $u(v)$ в фокальной диффракционной картине точечного источника света выражается формулой

$$
\begin{gathered}
u(v)=\left(c_{1}-c_{2}\right) a_{1} \frac{J_{1}\left(a_{1} v\right)}{v}+\left(c_{2}-c_{3}\right) a_{2} \frac{J_{1}\left(a_{2} v\right)}{v}+ \\
\ldots+\left(c_{n-1}-c_{n}\right) a_{n-1} \frac{J_{1}\left(a_{n-1} v\right)}{v}+c_{n} \frac{J_{1}(v)}{v},
\end{gathered}
$$

где $v=k \alpha ; k=\frac{2 \pi}{\lambda} ; \lambda-$ длина волны; $\alpha-$ расстояние точки в фокальной диффракционной картине от ее центра; $c_{1}, c_{2}, \ldots, c_{i}, \ldots, c_{n-1}$, $c_{n}, c_{n+1}=0$ - амплитудные коэффициенты пропускания компонентов фильтра; $a_{1}, a_{2}, \ldots, a_{i}, \ldots, a_{n-1}, a_{n}=a=1$ - радиусы концентрических окружностей, разделяющих компоненты фильтра; $J_{1}-$ функция Бесселя первого порядка.

Распределение интенсивности в диффракционной картине дается формулой

$$
I(v)=u(v) u^{*}(v) .
$$

Потребуем, чтобы в зонах диффракционной картины $v=v_{1}, v_{2}, \ldots$, $v_{n-1}$ свет гасился полностью, т. е. чтобы

$$
\begin{gathered}
u\left(v_{1}\right)=0 \\
u\left(v_{2}\right)=0 \\
\vdots \\
\vdots \\
u\left(v_{n-1}\right)=0 .
\end{gathered}
$$


Эти равенства образуют систему трансцендентных уравнений для тех значений величин $\left(c_{i}, a_{i}\right)$, при которых поставленное vсловие выполняется.

Напомним, что при отсутствии фильтра распределение интенсивности в диффракционной картине имеет вид $I_{0}=\frac{J_{1}^{2}(v)}{v^{2}}$, откуда следует, что первый минимум находится при $v=3,82$, а побочные максимумы при $v_{\mathrm{I}}=5,14 ; v_{\text {II }}=8,42$. Следовательно, если положим в (3) $0<v_{1}$, $v_{2}, \ldots, v_{n-1}<3,82$, то будем иметь сужение центрального максимума, а если возьмем $v_{1}=v_{\mathrm{I}}, v_{2}=v_{\mathrm{II}}$, то получим аподизацию.

Приведем далее относительные характеристики фильтров:

1) зональные дисперсионные коэффициенты $D_{2-4}^{r}, D_{5-9}^{r}$ и др., определяемые формулой

$$
D_{v^{\prime}-v^{\prime \prime}}^{r}=\frac{\int_{v^{\prime}}^{v^{\prime \prime}} I(v) v d v / \int_{0}^{\infty} I(v) v d v}{\int_{v^{\prime}}^{v^{\prime \prime}} I_{0}(v) v d v / \int_{0}^{\infty} I_{0}(v) v d v} ;
$$

2) полный энергетический коэффициент пропускания $\tau$ согласно формуле

$$
\tau=\frac{\int_{0}^{\infty} I(v) v d v}{\int_{0}^{\infty} I_{0}(v) v d v}=\sum_{i} c_{i}^{2}\left(a_{i}^{2}-a_{i-1}^{2}\right)
$$

3) интенсивность в фокусе $\frac{I(0)}{I_{0}(0)}$.

В случае двухкомпонентного фильтра система (3) сводится к одному уравнению. Если вместо амплитуды приравняем нулю интенсивность, то получим

$$
\begin{gathered}
I\left(c, a, v_{i}\right)=c_{1}^{2}\left[(1-c)^{2} a^{2} \frac{J_{1}^{2}(a v)}{v^{2}}+\right. \\
\left.+2 c(1-c) a \frac{J_{1}(v) J_{1}(a v)}{v^{2}}+c^{2} \frac{J_{1}^{2}(v)}{v^{2}}\right]\left.\right|_{v=v_{t}}=0
\end{gathered}
$$

где $\quad c=\frac{c_{2}}{c_{1}}, \quad a=a_{1}$.

Графический метод решения уравнения (6) позволяет легко найти фильтры с оптимальными параметрами $(c, a)$, дающими в диффракционной картине нулевую интенсивность в различных зонах $v=v_{i}$. На рис. 1 показаны линии нулевой интенсивности (ЛНИ) $I\left(c, a, v_{i}\right)=0$ на плоскости $(c, a)$. ЛНИ при $v_{i}=6 ; 7$ и 8 пересекаются в точке $c=0,31$; $a_{1}=0,55$. В области $5 \leqslant v \leqslant 9$ диффракционной картины с помощью такого двухкомпонентного амплитудного фильтра достигается значительная аподизация. На рис. 2 показана сетка линий постоянной дисперсии $D_{v^{\prime}-v^{\prime \prime}}^{r}=$ const, позволяющая оценить величину аподизации. Мы видим, что фильтр $c=0,31, a_{1}=0,55$ дает сильную аподизацию $D_{5-9} \approx$ $\approx 0,03$; но вместе с тем $D_{2-4}^{r}=1,7$, Согласно рис. 3 находим еще 


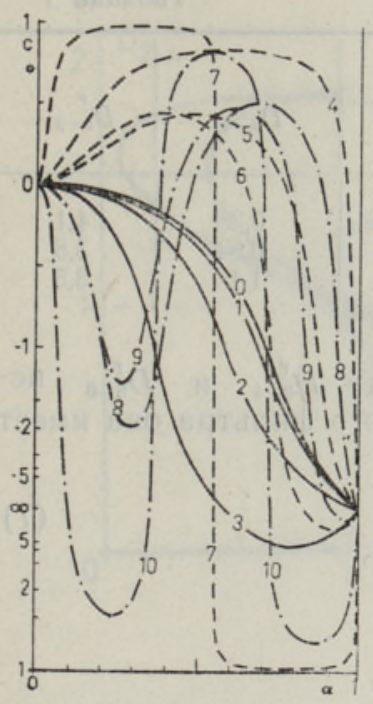

Рис. 1.

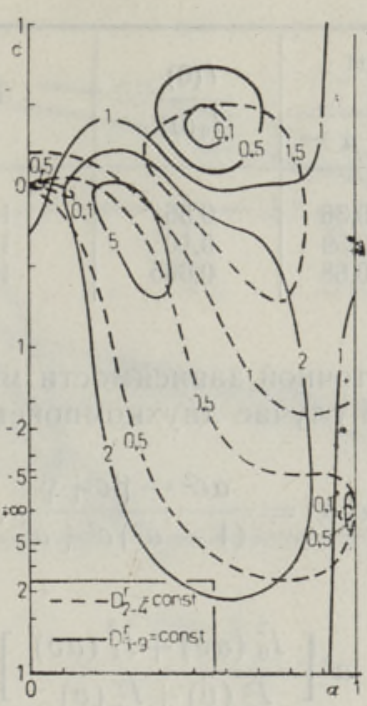

Рис. 2.

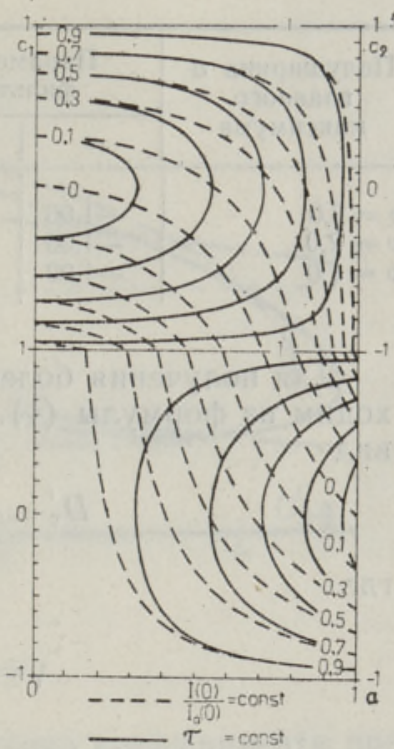

Рис. 3.

$\tau=0,37$ и $\frac{I(0)}{I_{0}(0)}=0,28$. Аналогичными свойствами обладает амплитудный фильтр с параметрами $c=0,42 ; a=0,55$. Характерно, что ЛНИ при $v_{i}=5 ; 6 ; 7 ; 8 ; 9 ; 10$, т. е. в области побочных максимумов, пересекаются друг с другом, а ЛНИ при $v_{i}=0 ; 1 ; 2 ; 3$, т. е. в области центрального максимума, - не пересекаются (см. рис. 1). Поэтому невозможно с помощью двухкомпонентных фильтров получить в области центрального максимума более одной зоны нулевой интенсивности. Bсе ЛНИ в области центрального максимума достигаются при $c<0$. Такой фильтр называется амплитудно-фазовым. Компонент с $c<0$ в таком фильтре изменяет не только амплитуду, но и фазу света на $\pi$. Из рис. 1 также видно, что ЛНИ при $v_{i}<3,8$ пересекаются с ЛНИ в области побочных максимумов $5 \leqslant v_{i} \leqslant 9$. Это означает, что амплитудно-фазовый фильтр, например, с параметрами $c=-0,81 ; a=0,55$ дает нулевую интенсивность как в области побочных максимумов, так и в области главного максимума. Это, однако, еще не означает, что одновременно подавляются побочные максимумы и сужается главный максимум. Здесь мы имеем всего лишь кажущуюся аподизацию, так как $D_{5-9}^{r}>1$, т. е. в области конечной ширины аподизации не происходит, наоборот, интенсивность увеличивается. Но главный максимум сужен и $D_{2-4}^{r}<1$.

Полученные результаты, дополненные данными табл. 1, выражают характерные черты взаимосвязи между сужением центрального максимума и аподизацией.

Из табл. 1 следует, например, что при сужении центрального максимума в 1,3 раза коэффициент дисперсии $D_{2-4}^{r}$ уменьшается примерно в 3 раза, но $D_{5-9}^{r}$ одновременно увеличивается примерно в 4 раза. Если же центральный максимум сужен в 2 раза, то $D_{2-4}^{r}$ уменьшается примерно в 1,2 раза, а $D_{5-9}^{r}$ увеличивается в 3,8 раза. 


\begin{tabular}{|c|c|c|c|c|c|c|}
\hline \multirow{2}{*}{$\begin{array}{c}\text { Полуширина } d \\
\text { главного } \\
\text { максимума }\end{array}$} & \multicolumn{2}{|c|}{$\begin{array}{c}\text { Параметры } \\
\text { фильтра }\end{array}$} & \multirow{2}{*}{$\frac{I(0)}{I_{0}(0)}$} & \multirow[t]{2}{*}{$\tau$} & \multirow{2}{*}{$D_{2-4}^{r}$} & \multirow{2}{*}{$D_{5-9}^{r}$} \\
\hline & $c$ & $a$ & & & & \\
\hline $\begin{array}{l}v=3,0 \\
v=2,0 \\
v=1,0\end{array}$ & $\begin{array}{l}-1,00 \\
-1,00 \\
-1,00\end{array}$ & $\begin{array}{l}0,36 \\
0,59 \\
0,68\end{array}$ & $\begin{array}{l}0,56 \\
0,10 \\
0,005\end{array}$ & $\begin{array}{l}1,0 \\
1,0 \\
1,0\end{array}$ & $\begin{array}{l}0,38 \\
0,84 \\
1,1\end{array}$ & $\begin{array}{l}4,1 \\
3,8 \\
3,5\end{array}$ \\
\hline
\end{tabular}

Для получения более точной зависимости между $D_{2-4}^{r}$ и $D_{5-9}^{r}$ исходим из формулы (4). В случае двухкомпонентного фильтра она имеет вид

$$
D_{v^{\prime}-v^{\prime \prime}}^{r}(c, a)=\frac{\alpha c^{2}-\beta c+\gamma}{\left(1-a^{2}\right) c^{2}+a^{2}}
$$

где

$$
\begin{gathered}
\gamma=-a^{2}\left[\frac{J_{0}^{2}(a v)+J_{1}^{2}(a v)}{J_{0}^{2}(v)+J_{1}^{2}(v)}\right]_{v^{\prime}}^{v^{\prime \prime}}, \\
\beta=2 \gamma+\left\{\frac{4}{v\left[J_{0}^{2}(v)+J_{1}^{2}(v)\right]} \sum_{i=0}^{\infty} a^{i+1} J_{i+1}(a v) J_{i}(v)\right\}_{v^{\prime}}^{v^{\prime \prime}}, \\
\alpha=1+\beta-\gamma .
\end{gathered}
$$

Согласно формуле (7) напишем выражения дисперсионных коэффициентов для зон $2-4$ и $5-9$, обозначив их через $D_{1}$ и $D_{2}$ :

$$
D_{2-4}^{r}(c, a)=D_{1} ; \quad D_{5-9}^{r}(c, a)=D_{2} .
$$

Исключая из этих равенств $c$, получим формулу, связывающую $D_{1}$ и $D_{2}$, в виде уравнения эллипса:

где

$$
a_{11} D_{2}^{2}+2 a_{12} D_{1} D_{2}+a_{22} D_{1}^{2}+2 a_{13} D_{2}+2 a_{23} D_{1}+a_{33}=0,
$$

$$
\begin{gathered}
a_{11}=\left(\alpha_{1} s-\gamma_{1} z\right)^{2}+\beta_{1}^{2} s z, \\
a_{12}=\left(\alpha_{1}+\alpha_{2}+\gamma_{1}+\gamma_{2}-1\right) s z-\alpha_{1} \alpha_{2} s-\gamma_{1} \gamma_{2} z, \\
a_{22}=\left(\alpha_{2} s-\gamma_{2} z\right)^{2}+\beta_{2}^{2} s z, \\
a_{13}=\left(\alpha_{2} \gamma_{1}-\alpha_{1} \gamma_{2}\right)\left(\alpha_{1} s-\gamma_{1} z\right)+\frac{\beta_{1} \beta_{2}}{2}\left(\alpha_{1} s+\gamma_{1} z\right)-\frac{\beta_{1}^{2}}{2}\left(\alpha_{2} s+\gamma_{2} z\right), \\
a_{23}=\left(\alpha_{2} \gamma_{1}-\alpha_{1} \gamma_{2}\right)\left(\gamma_{2} z-\alpha_{2} s\right)+\frac{\beta_{1} \beta_{2}}{2}\left(\alpha_{2} s+\gamma_{2} z\right)-\frac{\beta_{2}^{2}}{2}\left(\alpha_{1} s+\gamma_{1} z\right), \\
a_{33}=\left(\alpha_{2} \gamma_{1}-\alpha_{1} \gamma_{2}\right)^{2}-\beta_{1} \beta_{2}\left(\alpha_{2} \gamma_{1}+\alpha_{1} \gamma_{2}\right)+\alpha_{2} \beta_{1}^{2} \gamma_{2}+\alpha_{1} \beta_{2}^{2} \gamma_{1} \\
\left(s=a^{2} ; \quad z=1-a^{2}\right) .
\end{gathered}
$$

На рис. 4 приведены эллипсы для некоторых значений $a$. Совокупность всех возможных эллипсов определена в четырех областях:
1) $D_{2-4}^{r}<1$ и $D_{5-9}^{r}>1$;
2) $D_{2-4}^{r}>1 \quad$ и $\quad D_{5-9}^{r}<1$;
3) $D_{2-4}^{r}<1 \quad$ и $\quad D_{5-9}^{r}<1$;
4) $D_{2-4}^{r}>1$ и $D_{5-9}^{r}>1$. 


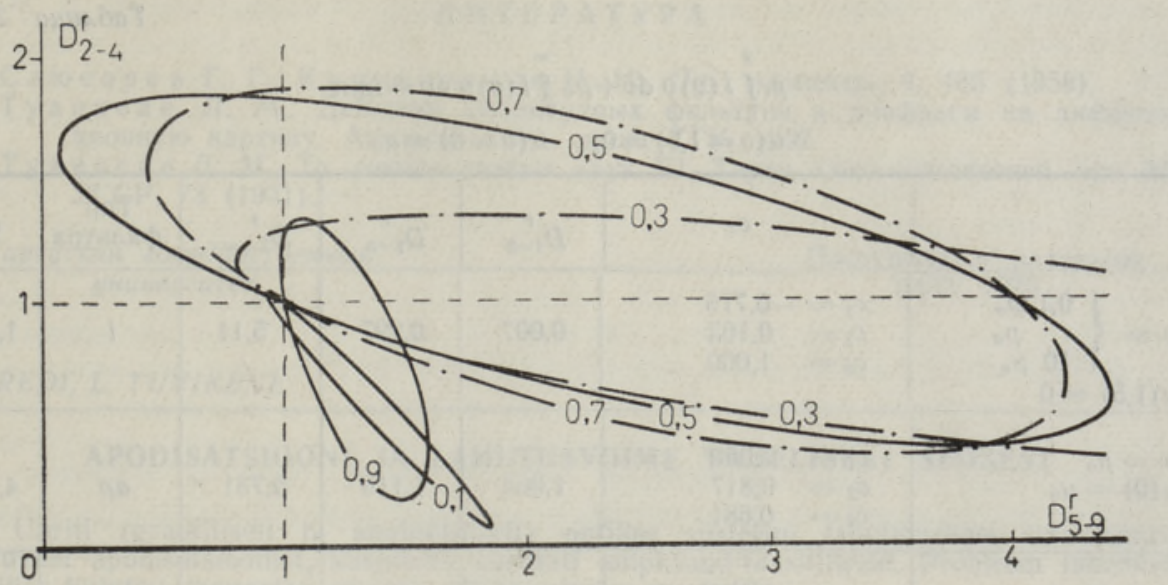

Рис. 4 .

Точкам каждого эллипса соответствуют значения коэффициента пропускания $c$. Зависимость между $D_{2-4}^{r}$ и $D_{5-9}^{r}$, соответствующую случаям 1 и 2 , назовем нормальной, а зависимость, соответствующую случаям 3 и 4, - аномальной. Нормальная и аномальная зависимости изображаются на рис. 4 соответствующими дугами эллипсов. Если расширить зону побочных максимумов и вместо $5-9$ взять $5-\infty$ (или $3-\infty$ ), то зависимость между $D_{2-4}^{r}$ и $D_{5-\infty}^{r}$ может стать нормальной. Для доказательства последнего утверждения обобщим метод нулевых зон интенсивности (формулы (3) и (6)). Вместо условия (3) потребуем минимума интегральной интенсивности диффракционной картины от $v^{\prime}$ до $v^{\prime \prime}$ :

$$
\begin{gathered}
\int_{v^{\prime}}^{v^{\prime \prime}} I(v) v \mathrm{~d} v=\min \\
\text { при } \quad u\left(v=v_{0}\right)=u_{0} .
\end{gathered}
$$

Сформулируем условие минимума вместо (12) в более общей форме:

$$
p_{l} \cdot \int_{v_{0}^{\prime}}^{v_{0}^{\prime \prime}} I(v) v \mathrm{~d} v+p_{a} \cdot \int_{v^{\prime}}^{v^{\prime \prime}} I(v) v \mathrm{~d} v=\min ,
$$

где первый интеграл означает интегральную интенсивность диффракционной картины в области центрального максимума от $v_{0}^{\prime}$ до $v_{0}^{\prime \prime}$, а второй - в области побочных максимумов от $v^{\prime}$ до $v^{\prime \prime} ; p_{l}$ и $p_{a}-$ некоторые численные множители.

В табл. 2 приведены для различных значений $p_{l}$ и $p_{a}$ (и различных форм условия (13)) значения коэффициентов пропускания $c_{i}$ трехкомпонентного фильтра, минимизирующие выражение (14). Там же приведены значения других характеристик. Значения радиусов компонент приняты равными $a_{1}=0,577 ; a_{2}=0,816 ; a_{3}=1,000$, а пределы интегралов положены $v_{0}^{\prime}=1 ; v_{0}^{\prime \prime}=2 ; v^{\prime}=3 ; v^{\prime \prime}=\infty$.

Из табл. 2 видно, что при дополнительном условии в виде $u(v=1,5)=0$ полуширина $d$ центрального максимума не зависит от отношения $\begin{gathered}p_{l} \\ p_{a}\end{gathered}$ (в пределах от 0,1 до 10 ). Без этого условия получа- 


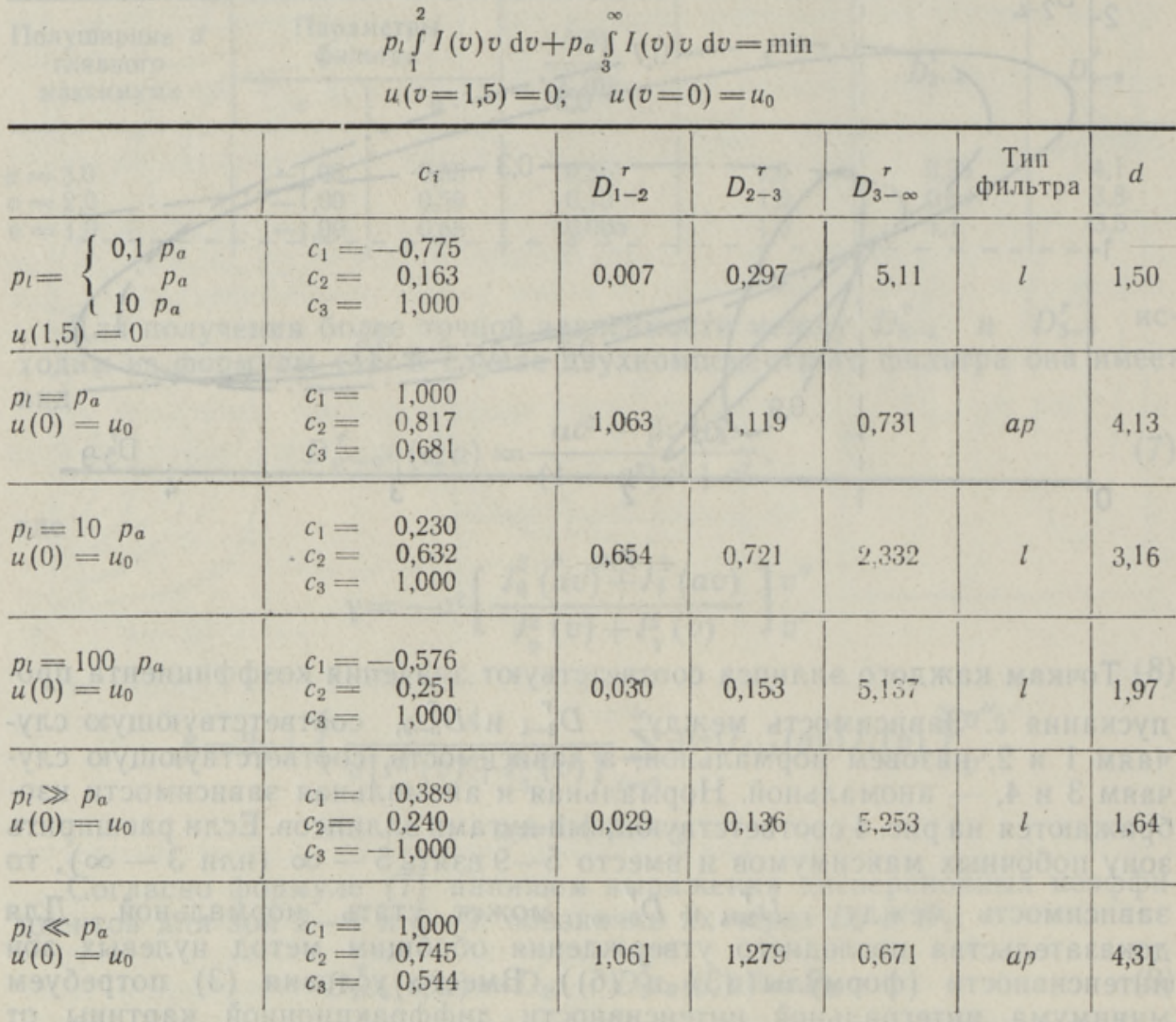

$l$ - фильтр, сужающий центральный максимум,

$a p$ - аподизирующий фильтр,

$d$ - полуширина центрального максимума.

ются: 1) при $\frac{p_{l}}{p_{a}}=0 ; 1-$ аподизирующие фильтры, у которых $D_{3-\infty}^{r}<1 \quad\left(D_{1-2}^{r}>1 ; \quad D_{2-3}^{r}>1\right) \quad$ и $\left.d>3,8 ; 2\right)$ при $\frac{p_{l}}{p_{a}}=10 ; 100 ; \infty-$ фильтры, сужающие центральный максимум, у которых $D_{1-2}^{r}<1$;

$D_{2-3}^{r}<1 \quad\left(D_{3-\infty}^{r}>1\right)$ и $d<3,8$.

Таким образом, мы показали, что взаимосвязь между $D_{1-2}^{r}$ (также $D_{2-3}^{r}$ ) и $D_{3-\infty}^{r}$ является нормальной. Влияние амплитудного фильтра на диффракционную картину круглого отверстия может быть двух типов: 1) либо происходит аподизация с одновременным уширением центрального максимума, 2) либо происходит сужение центрального максимума с одновременным возрастанием побочного максимума. 
ЛИТЕРАТУРА

1. С люсарев Г. Г., Куликовская Н. И., Опт. и спектр., 4, 486 (1958).

2. Т у викене Л. М., Действие амплитудных фильтров и диафрагм на диффракционную картину, Автореф. канд. дисс., ТГУ, 1962.

3. Т увикене Л. М., Тр. секции точных наук VI, Тарту, О-во естествоисп. при АН ЭССР, 73 (1971).

Тартуский государственный университет

Поступила в редакцию 28/IV 1975

V. REDI, L. TUVIKENE

\section{APODISATSIOONI JA LAHUTUSVÖIME VAHELISEST SEOSEST}

Uuriti (graafiliselt ja analüütiliselt) optilise süsteemi lahutusvõime suurenemise sõltuvust apodisatsioonist, kusjuures kasutati amplituud-faasifiltreid. Probleem lahendati optilise kujutise tsonaalse energia minimeerimise meetodil.

\section{REDI, L. TUVIKENE}

\section{ON THE INTERRELATIONSHIP BETWEEN APODISATION AND RESOLUTION}

The paper deals with the problem of magnifying the resolution of an optical system, depending on apodisation. The dependence has been studied graphically and analytically, applying phase-amplitude filters. The problem has also been solved as a minimum task. 